\title{
Orientación de las competencias socioemocionales de los alumnos de Educación Secundaria en contextos multiculturales
}

\section{Elvira Repetto Talavera, Mario Pena Garrido, Maria José Mudarra, Maite Uribarri}

Departamento MIDE,

Universidad Nacional de Educación a Distancia, Madrid

España

erepetto@edu.uned.es

mpena@edu.uned.es

mjmudarra@edu.uned.es 


\section{Resumen}

Uno de los objetivos de la Orientación Educativa es el desarrollo social y el aprendizaje de los alumnos de Educación Secundaria en los contextos multiculturales a través del incremento de sus competencias socioemocionales y mediante la intervención de programas de orientación. El Programa de Orientación en las Competencias Socio-emocionales (POCOSE) que se propone, mejora no sólo el proceso de aprendizaje y el éxito académico de estos alumnos, sino que también favorece la integración escolar y social de los mismos y con ello, ejerce una función preventiva ante futuros "fracasos escolares" y otros factores de riesgo, tal como el absentismo, el abandono escolar o la violencia (bullying).

La amplia diversidad cultural existente en nuestro sistema educativo requiere identificar qué competencias socioemocionales se relacionan positivamente con un óptimo aprendizaje y una mejor adaptación socioeducativa de los/las estudiantes de secundaria en los contextos multiculturales, así como identificar las diferencias culturales existentes en el proceso de enseñanza y aprendizaje de estas competencias.

Asimismo, es necesario disponer de programas de orientación y formación en competencias socioemocionales, validados científicamente, ya que son un medio adecuado para la mejora del aprendizaje y la adaptación socioeducativa del alumnado de secundaria.

Palabras Clave: orientación, intervención de programas, competencias socio-emocionales, multiculturalidad 


\section{Introducción}

Dentro del marco de la Orientación de la persona a lo largo de la vida, y concretamente en el ámbito de la Orientación Escolar, uno de los objetivos fundamentales es el desarrollo social y el aprendizaje de los alumnos en los contextos multiculturales, a través de la mejora de sus competencias socioemocionales mediante programas de intervención, como por ejemplo, el Programa de Orientación en las Competencias Socioemocionales (POCOSE), el cual se inserta en el Proyecto I+D, "Orientación y desarrollo de competencias socio-emocionales a través de las prácticas en empresa", financiado por el Ministerio de Educación, (SEJ200407648/EDUC), y que dirije Repetto (2005-2007).

La orientación e intervención mediante programas de competencias socio-emocionales mejorará no sólo el proceso de aprendizaje y el éxito académico de estos alumnos, sino que también favorecerá la integración escolar y social de los mismos y con ello, ejercerá una función preventiva ante futuros "fracasos escolares" y otros factores de riesgo, tal como el absentismo, el abandono escolar o la violencia (bullying).

En este artículo, se expone en primer lugar, la situación de la inmigración en Europa y, concretamente en España, así como las intervenciones desde la orientación intercultural; en segundo lugar, se hace referencia a las competencias socio-emocionales y sus beneficios educativos en los contextos multiculturales; a continuación, se destaca la relevancia de estas competencias en el ámbito de la Orientación Profesional, y posteriormente se presentan los grupos nacionales e internacionales que están trabajando en esta temática; finalmente se añaden unas conclusiones finales.

\section{La inmigración y la orientación intercultural}

La construcción de un nuevo marco europeo multicultural abierto al crecimiento de los movimientos migratorios así como las respuestas insolidarias, racistas, e intolerantes, junto con el fracaso académico de algunos colectivos, son factores que nos obligan a implicarnos en el reto que supone el pluralismo cultural y las respuestas educativas correspondientes. El desafío con el que nos encontramos es el de asumir la "mundialización" de la cultura mientras se preserva y enriquece la propia identidad cultural. Según indican Repetto y Pena (2006a) el 
contacto con grupos y etnias dispares obliga al reconocimiento de la multiplicidad de enfoques y, por tanto, al desarrollo de instituciones educativas dialogantes y democráticas, centradas en la promoción de la convivencia y la potenciación (empowerment) de cada uno de los estudiantes y de sus enfoques culturales.

Asimismo, la inmigración se ha convertido en un fenómeno característico de nuestra sociedad, como lo demuestra el hecho de que cada vez es más numeroso el grupo de alumnos extranjeros en las aulas españolas. De acuerdo con el Ministerio de Educación (2006), en España, el número de alumnos extranjeros en enseñanzas no universitarias en el curso 2005-2006 se estima en 529.461, unos 80.000 más que el año anterior y unos 400.000 alumnos más que hace diez años. De esta forma, el alumnado extranjero representa el 6,5\% del total de alumnado no universitario, aunque con diferencias significativas entre las Comunidades Autónomas: las que cuentan con mayor número de alumnos extranjeros por 1.000 alumnos matriculados en Educación Infantil, Primaria y Secundaria Obligatoria son la Rioja (112,5), Illes Balears $(100,1)$, Murcia $(97,2)$, Navarra $(88)$ y Madrid $(87,7)$; este dato confirma la trayectoria del curso 2004-2005 en el que las comunidades autónomas con mayores porcentajes eran Illes Balears (11,1\%), Madrid (10,7\%) y La Rioja (9,6\%). También existen diferencias considerables en su distribución por la titularidad del centro donde se escolarizan: en los centros públicos, el número de alumnos extranjeros por cada 1.000 alumnos matriculados es de 80,6, más del doble que en los centros privados $(32,5)$. En cuanto a su nacionalidad de origen, sigue destacando el alumnado procedente de América del Sur, con 230.381 alumnos (Ecuador y Colombia fundamentalmente), seguido de Europa con 144.508 alumnos (Rumanía fundamentalmente) y, en tercer lugar, África con 103.112 alumnos (Marruecos fundamentalmente). Ante este nuevo escenario, la educación debe atender, de una parte, la socialización del individuo mediante la recepción de los valores imperantes en su sociedad, y de otra, la autonomía de dicho sujeto de modo que sea capaz de distanciarse de condicionamientos culturales (Repetto y Pena, 2006b).

Si observamos la situación escolar de los alumnos de Educación Secundaria se advierten diferencias existentes entre los jóvenes de diversas culturas, constatándose mayores índices de fracaso escolar entre la población inmigrante. Según Repetto (2006a), se entiende por fracaso escolar el desajuste negativo entre la capacidad real de un alumno y su rendimiento escolar valorado académicamente. Aproximadamente un 30\% de tales fracasos escolares se deben a déficits de competencias socioemocionales y un $20 \%$ a la falta de apoyo por parte del contexto educativo 
y familiar. Efectivamente, Tornos y Aparicio (2005) señalan que los adolescentes inmigrantes abandonan antes los estudios que los españoles, ya que, de hecho, sólo un 14,7\% de los hijos de inmigrantes cursa bachillerato superior, un porcentaje que representa algo menos de la mitad que el de los estudiantes españoles, que ronda el 37\%. Las diferencias son mayores cuando hablamos de la Universidad, ya que tan sólo un 13\% de los hijos de inmigrantes alcanzan estos estudios frente a un 39\% de los españoles. Los inmigrantes prefieren la opción de la formación profesional, a la que acude el 17,4\%. Asimismo, los alumnos extranjeros manifiestan dificultades para finalizar sus estudios como lo demuestra el hecho de que el 53\% de ellos continúa estudiando la ESO aún habiendo sobrepasado la edad de 16 años. Si tenemos en cuenta las diferentes nacionalidades observamos que los dominicanos y peruanos que cursan el Bachillerato triplican en proporción a los marroquíes; por el contrario, los de origen magrebí casi duplican a los otros dos grupos en los estudios de formación profesional y son los que sufren más fracaso escolar. Se constata de esta forma, una situación de riesgo tanto de fracaso escolar como de desadaptación socioeducativa.

La orientación intercultural en los centros educativos, según indican Repetto y Pena, (2006b) es necesaria para favorecer la motivación y mejorar los aprendizajes de todos los alumnos, particularmente los procedentes de otras culturas que pueden tener experiencias de desesperanza aprendida y creencias erróneas que podrían pervivir durante toda su vida adulta, como por ejemplo, pensar que el currículo académico es irrelevante para su vida laboral. Además, estos alumnos suelen manifestar falta de confianza en sus capacidades, agudizada por tratamientos discriminatorios. Por otra parte, el desarrollo cognitivo, tiene lugar en un determinado "nicho cultural", (Repetto, 2006b), por lo que debe valorarse en función de su grado de adaptación a las exigencias del contexto, de modo que las interacciones entre los alumnos y su contexto multicultural generan cambios en su desarrollo cognitivo. Actualmente, se cuestiona un modelo único de desarrollo cognitivo para adquirir y utilizar los conocimientos, primando el factor cultural que incide en la producción de un conocimiento en un medio cultural. Ahora bien, si como señala Ortega (1996), toda educación de naturaleza multicultural ha de generar diálogo, encuentro y convivencia y propugnar la erradicación de actitudes y conductas intolerantes caracterizadas por la exclusión de las personas por motivo de su raza o su lengua, es precisa la adquisición de un repertorio de competencias socioemocionales para participar efectivamente en tal convivencia. De este modo, no se trata tanto de proponer programas independientes para prevenir la violencia, el abandono escolar, y otros factores de riesgo, sino intervenciones holísticas, coordinadas, que promuevan aprendizajes socia- 
les y emocionales e incidan en las variables mediadoras del éxito académico -en sentido amplio, esto es, actitudes, conducta y resultados escolares positivos (Zins et al., 2004)- como la motivación, autorregulación y el autoconocimiento emocional. Por tanto, la orientación intercultural puede mejorar estos factores negativos mediante: la demostración de la relevancia que el aprendizaje formal tiene para la vida laboral; la clarificación de las relaciones entre la educación y el logro de objetivos profesionales; la percepción de las metas educativas que se les ofrece; la potenciación del yo y la superación de los estereotipos relacionados con la etnia, el género, la clase social o la cultura. En particular, los programas de orientación intercultural para el desarrollo personal y social se caracterizan, entre otros aspectos, por desarrollar la autoestima, la implicación en actividades significativas y reducir la posibilidad de exclusión social (Repetto y Pena, 2006a). Teniendo en cuenta que la Orientación intercultural en contextos escolares pretende fortalecer la autoestima, el auto-concepto positivo y el desarrollo de relaciones interpersonales y respeto mutuo, es decir, el desarrollo personal y social de los alumnos, uno de los enfoques de intervención más pertinente es el de competencias y en particular centrado en las de carácter socio-emocional (Repetto y Pérez-González, en prensa).

\section{Las competencias socio-emocionales y sus beneficios educativos en contextos multicultu- rales.}

A nivel internacional se ha incrementado el interés por el desarrollo de las competencias en ámbitos académicos, tal y como se aprecia en el proyecto Tuning Educational Structures in Europe, cuyo objetivo es la formación de los estudiantes universitarios en competencias genéricas y específicas, destacando entre las primeras las competencias socioemocionales. Tales competencias son especialmente relevantes en el ámbito educativo como señalan Weissberg y Greenberg (1998) y CASEL (2003). No obstante, el factor cultural ha sido poco investigado desde el punto de vista de su incidencia en el nivel de competencias socioemocionales; en el centro educativo se evidencia la necesidad, pero también la posibilidad, de socializar al alumnado extranjero al mismo tiempo que se favorecen los aprendizajes escolares de todos sus alumnos. Una de las estrategias de intervención la encontramos en los Programas para la Tolerancia y la Prevención de la Violencia en la Juventud (Díaz-Aguado, 1996) y el programa Convivir es vivir (MEC, 1997) -iniciativa puesta en marcha por la Comunidad de Madrid- dirigido éste último a jóvenes y adolescentes de 12 a 18 años, con el objetivo de prevenir la violencia en los centros y fomentar una educación para la convivencia. 
Más allá de la inteligencia académica, el desarrollo de las competencias socio- emocionales permite abordar el problema del fracaso escolar como condición sociopersonal que se manifiesta en la incapacidad del individuo para alcanzar los objetivos educativos propuestos por un Sistema o Centro escolar, para un determinado nivel curricular (García, 2003).

El análisis de la literatura existente permite constatar algunas de las razones que justifican la intervención en torno a las competencias socioemocionales (Pena y Repetto, 2006). Dentro de las aportaciones de autores extranjeros, destacamos, por su difusión, a Goleman (1996), quien basándose en investigaciones americanas, pone de manifiesto el bajo nivel de competencia emocional de los jóvenes de esta década, los cuales se caracterizan por lo que él denomina "analfabetismo emocional". En esta misma línea se expresan otros autores (Graczyk, Weissberg, Payton, Elias, Greenberg y Zins, 2000; Dryfoos, 1997; Scales y Leffert, 1999), que reclaman atención sobre el conjunto de comportamientos desadaptativos que presentan los jóvenes, tales como conductas agresivas y peleas, posesión de armas, consumo de drogas y alcohol, conducta sexual inadaptada o intentos de suicidio. Del mismo modo, Steiner (1998) establece que cuando somos emocionalmente educados podemos manejar situaciones emocionales difíciles que a menudo llevarían a peleas, enfados o respuestas hirientes. En España, algunos autores han puesto de relieve la importancia de la educación emocional como una forma de prevención para minimizar la vulnerabilidad a las disfunciones y prevenir su ocurrencia (Bisquerra, 2000; Ibarrola, 2004); por otro lado, Fernández-Berrocal y Extremera (2002) abordan la cuestión del cociente intelectual como un mal predictor de éxito en la vida, ya que la inteligencia académica no es suficiente para alcanzar el éxito profesional, a la vez que tampoco garantiza la satisfacción en el mundo de las relaciones personales; todo ello, sumado a un conjunto de situaciones sociales hace necesaria la intervención socio-emocional en el ámbito educativo (Álvarez, 2001).

El papel de las competencias socioemocionales como predictor de la variable "rendimiento escolar" y como factor de mejora del aprendizaje ha sido puesto de relieve en diversas investigaciones nacionales e internacinales (Extremera y Fernández-Berrocal, 2004). De acuerdo con Schutte, Malouff, Hall et al. (1998) las puntuaciones en instrumentos que miden competencias socioemocionales predicen significativamente la nota media de los alumnos universitarios a final de curso; asimismo, Parker, Summerfeldt, Hogan y Majeski (2004) utilizaron la variable “competencias socioemocionales" como predictora para incluir a los alumnos en el grupo de alto y bajo rendimiento académico; según este último estudio, en líneas generales, las competencias socioemocionales identifican a los estudiantes de primer año que tienen éxito académico al fina- 
lizar el semestre; en concreto, el $82 \%$ de los estudiantes con alto rendimiento académico y el 91\% de los alumnos con bajo nivel académico fueron correctamente identificados y agrupados en función de sus puntuaciones en la variables competencias socioemocionales. En nuestro país, Fernández-Berrocal, Extremera y Ramos (2004) se centran en estudiar el efecto mediador que una buena salud mental ejerce sobre el rendimiento medio escolar de los estudiantes; en general los resultados de este estudio, tomados en su conjunto, permitieron vislumbrar que aquellos alumnos con mayor desarrollo de competencias socioemocionales presentan mejor equilibrio psicológico, que a su vez, está relacionado con y afecta al rendimiento académico. En este sentido, los resultados indican que en los adolescentes con problemas de aprendizaje, las competencias socioemocionales pueden actuar como un moderador de los efectos de las capacidades cognitivas sobre el rendimiento académico (Petrides, Frederickson y Furnham, 2004). No obstante, el peso de los aprendizajes socioemocionales en el logro académico depende, como señalan Zins et al. (2004), de diversos factores como la forma en que se define y evalúa dicho logro, el ambiente en que se desarrollan las experiencias de aprendizaje, más o menos tradicional o motivador, el grado en que los programas se contextualizan específicamente en función del centro educativo o se vinculan con sus iguales o figuras de referencia adultas. Todo ello debe ser tenido en cuenta.

Por tanto, parece existir suficiente base científica para avalar las relaciones entre los aprendizajes socioemocionales y el «éxito académico » y en último término, el « éxito en la vida » (Zins, Weissberg, Wang y Walberg. 2004). También la conducta prosocial en clase se relaciona significativamente con resultados intelectuales y académicos positivos (DiPerna y Elliot, 1999; Haynes, Ben-Avie y Ensign, 2003; Malecki y Elliott, 2002; Pasi, 2001). A la inversa, la conducta antisocial que a menudo manifiestan alumnos de bajo rendimiento, también está ampliamente documentada (Hawkins, Farrington y Catalano, 1998), de modo que este tipo de intervenciones resulta particularmente eficaz con jóvenes de clase media-baja más propensos a implicarse en actividades violentas, consumo de alcohol y actividades sexuales que derivaban en embarazos no deseados.

Por todo esto, es necesario fomentar las intervenciones psicopedagógicas para el desarrollo de estas competencias a lo largo de la vida, centradas en el diseño y aplicación de programas de educación emocional y complementadas, en la medida de lo posible, con otras estrategias como la infusión curricular de la enseñanza de las competencias socioemocionales (Pena y Repetto, 2006; Zeiner, Roberts y Matthews, 2002). De hecho, el 34\% de los progra- 
mas revisados, incluyen métodos para promover la integración del aprendizaje de competencias socioemocionales en el currículo escolar y en las estrategias docentes en cada una de las áreas (Zins, Weissberg, Wang y Walberg, 2004). Mediante la aplicación de estos programas, los alumnos no sólo amplían su vocabulario emocional, sino que aprenden a emplear estrategias de afrontamiento ante situaciones emocionalmente difíciles, alcanzando el autocontrol emocional, de modo que manejen adecuadamente las emociones e impulsos conflictivos (Vallés y Vallés, 2000). Estas estrategias autorreguladoras son muy útiles en el contexto escolar cuando, por ejemplo, se acometen tareas académicas, a la vez que resultan eficaces en otros contextos no escolares -familiar y social- (Torres, 1996), con lo que logramos que se transfieran las competencias socio-emocionales asociadas a la inteligencia emocional del ámbito académico a otros ámbitos, como el personal y el social.

Finalmente, otros beneficios educativos de este tipo de intervenciones, y de las competencias socioemocionales en general, consisten en prevenir factores de riesgo en el aula, disminuyendo el número de expulsiones de clase y el índice de agresiones, y en mejorar las califícaciones académicas y el desempeño escolar (CASEL, 2003), así como el incremento de los niveles de bienestar y ajuste psicológico, y la satisfacción de las relaciones interpersonales de los alumnos (Extremera y Fernández-Berrocal, 2004). Así, se incrementan las oportunidades de estos alumnos para participar en actividades prosociales y obtener recompensas eficaces por su participación.

\section{Relevancia de las competencias socio-emocionales en contextos profesionales: un desafío para la Orientación Profesional.}

Las sociedades modernas demandan profesionales equipados con una amplia variedad de competencias, entre las que destacan, cada vez más, aquellas que no se circunscriben exclusivamente a las tareas inherentes a cada puesto de trabajo, sino que se refieren a la forma de trabajar, la actitud hacia el trabajo, la actitud hacia los demás, la cualidad y calidad de las relaciones humanas, la flexibilidad o la capacidad de adaptación. Más allá del saber o del saber hacer, se exige querer hacer, saber ser y saber estar, considerando aspectos axiológicos implícitos en la forma de percibir y vivir con los otros en un contexto social. De hecho, se ha demostrado que este tipo de competencias socio-emocionales facilitan la inserción laboral de los estudiantes universitarios y, en general, puede considerarse que potencian la empleabiliadad (Caruso y Wolfe, 2001; Hettich, 2000). 
En contextos organizacionales, junto a las habilidades cognitivas, las competencias socioemocionales se estiman cada vez más relevantes para lograr elevadas cotas de desempeño y desarrollo profesional (Izquierdo, 2001). El desarrollo de tales competencias socioemocionales juega un papel importante para reforzar el éxito individual en un contexto organizacional tal como evaluaron Dulewicz y Higgs, (1999). La necesidad de este tipo de competencias abarca un amplio abanico de tareas que van desde la dirección de grupos, el trabajo en equipo, la tolerancia al estrés laboral, la capacidad negociadora, la resolución de conflictos, la planificación de la propia carrera profesional, la motivación hacia el trabajo, hacia otros o el afrontamiento de situaciones críticas. No obstante, además del éxito individual, es preciso saber trabajar en equipo, cooperar, coordinarse con otros, en definitiva, poner en práctica competencias socioemocionales que faciliten y optimicen tanto el trabajo conjunto como la calidad de las relaciones (Charbonneau y Nicol, 2002; Shutte et al. 2001).

Entre estas interacciones mutuas entre el individuo y su entorno laboral, características del desarrollo profesional actual, cabe destacar las influencias mutuas entre las demandas del individuo, el ambiente organizacional y la efectividad, demostrándose la relación directa entre el clima, la cultura y el sistema organizacional y la efectividad en diferentes áreas como la selección de personal, el trabajo en equipo, el compromiso moral y la salud del empleado, la innovación, la productividad, la eficiencia, los niveles de ventas e ingresos, la calidad del servicio y la lealtad del cliente (Boyatzis et al. 2000; Chermiss y Goleman, 2001). Así se han evidenciado relaciones importantes entre las competencias socio-emocionales y el desarrollo de la carrera (Repetto y Pérez-González, en prensa; Caruso y Wolfe, 2001), el desempeño laboral (Bachman et al. 2000) y el liderazgo efectivo (Segal, 2002).

Las competencias socio-emocionales influyen también en el proceso de adaptación del individuo al ambiente laboral -el manejo del estrés y la presión ocupacional- considerándose criterios básicos en la predicción del comportamiento laboral y el éxito ocupacional (Bar-On et al, 2000; Wong y Law, 2002). Tales aspectos son particularmente importantes en los contextos multiculturales donde la carencia o insuficiencia de este tipo de competencias supondría un factor de riesgo en los procesos de inserción y desarrollo profesional que agravaría aún más el riesgo de exclusión social.

En consecuencia, tanto los procesos de inserción como las diversas transiciones profesionales exigen la propuesta de programas de Orientación, dirigidos al desarrollo de las com- 
petencias socioemocionales capaces de afrontar los efectos de la inestabilidad laboral, el desempleo, las exigencias de movilidad profesional, el complejo entramado de relaciones sociolaborales y la calidad de vida en el trabajo.

Uno de los programas más relevantes en este ámbito, y dentro de la Orientación Profesional, es el Programa de Orientación en las Competencias Socioemocionales (POCOSE), que se inserta en el Proyecto I+D, "Orientación y desarrollo de competencias socio-emocionales a través de las prácticas en empresa", financiado por el Ministerio de Educación, (SEJ200407648/EDUC), y que dirije Repetto (2005-2007).

El programa POCOSE tiene como objetivo principal la orientación y formación en competencias socioemocionales, aplicándose en el marco del Practicum y de las Prácticas en Empresa, y de este modo, favorecer el desarrollo personal y la inserción laboral del colectivo de estudiantes y recién titulados. Se compone de siete módulos, cada uno de los cuales desarrolla una de las siguientes competencias: autoconocimiento emocional, empatía, regulación emocional, motivación, asertividad, trabajo en equipo y resolución de conflictos; en cada uno de los módulos se especifican los objetivos, contenidos, dinámica de desarrollo, secuencia de las sesiones, materiales empleados y bibliografía más relevante. Asimismo, estos módulos constan, por un lado, de un libro del profesor en el que se ofrece una presentación general del módulo, además de una guía didáctica en la que se explica el desarrollo de cada sesión junto con los materiales gráficos, audiovisuales, informáticos y otra serie de documentos necesarios para su desarrollo; por otro lado, se cuenta con un documento que recoge los materiales de trabajo del alumno necesarios para el desarrollo de las sesiones.

Con el objetivo de validar científicamente este programa, se cuenta con un inventario de competencias socio-emocionales (ICOSE, pendiente de publicación), que se aplica como medida pre y post test, y con dos cuestionarios a través de los cuales se evalúa la importancia de estas competencias en estudiantes de Ciclos Formativos y de Universidad así como el grado de presencia que dichas competencias tienen en sus respectivas ramas de formación (Repetto, Beltrán, Garay-Gordovil y Pena, 2006).

Con este programa, y con otros que puedan surgir en investigaciones futuras, la Orientación Profesional superaría el desafío de convertirse en un auténtico recurso social para 
afrontar las múltiples elecciones y decisiones ocupacionales que cada individuo habrá de adoptar a lo largo de su vida.

El desarrollo de las competencias socio-emocionales en los grupos nacionales e internacionales.

En nuestro país, la investigación sobre programas de competencias socioemocionales es reciente, sin embargo, podemos destacar la línea de investigación sobre competencias dirigida por la Dra. Repetto (UNED, Facultad de Educación), centrada, por un lado, en la realización del Proyecto I+D, Orientación y desarrollo de competencias socio-emocionales a través de las prácticas en la empresa y en la Universidad (SEJ2004-07648/EDUC, 2005-2007), financiado por el Ministerio de Educación español, y por otro, en la dirección del Centro de Investigación Orientación en Competencias para la Inserción y el Desarrollo Profesional (2004).

Entre los grupos nacionales que han investigado sobre las competencias socioemocionales destaca el dirigido por Guil (2006), cuyo proyecto de investigación se enmarca en el ámbito de la Psicología ya que se centra en el análisis psicológico del constructo de inteligencia emocional y de alternativas para su medida. Por otra parte, nuestra propuesta hunde sus raíces en la Pedagogía, dado que su finalidad es la elaboración de un programa de formación en competencias socio-emocionales dirigido a cubrir una necesidad existente en nuestros centros educativos. Asimismo cabe destacar los trabajos de Pascual y Cuadrado (2001), Vallés y Vallés (1999), Vallés (2003) o Güell Barceló y Muñoz (2000, 2003), así como el trabajo desarrollado por el GROP de Barcelona (Álvarez, 2001). Otras investigaciones consideran diversos factores entre los que se incluyen las competencias sociales y emocionales, como las de Garaigordobil, (2005), Arón y Milicia (1996), De la Cruz y Mazaira (1997), Traveset (1999), Blasco, Bueno, Navarro y Torregrosa (2002), Espejo, García-Salmones y Vicente (2000), Salvador (2000), Salmurri y Blanxer (2002), Ibarrola y Delfo (2003a, 2003b), Renom (2003), y López Cassà (2003). No obstante, al revisar las variables estudiadas en relación con el tema y la existencia de diferentes programas e instrumentos de intervención y evaluación en nuestro país, apreciamos que suelen insistir en el desarrollo de los mismos conocimientos, habilidades y/o destrezas con leves matices pero muy pocos se aplican de forma científica. Apenas se recurre al uso de diseños experimentales y psicométricos fiables, ni se especifica la teoría en la que se sustenta la intervención, ni se identifica el contexto educativo y sociocultural al que va destinado, contribuyendo notablemente a la confusión cuando se trata de evaluar real- 
mente los logros alcanzados (Zeidner, Roberts y Matthews, 2002). Sin embargo, citamos los trabajos del GROUP (Bisquerra, 2004) que han iniciado el proceso de evaluación de estos programas, cuyos resultados aún no están disponibles en la literatura especializada. Por todo ello, abogamos por emprender investigaciones rigurosas de evaluación de los programas e instrumentos de formación en competencias socio-emocionales (Bisquerra, 2004; Filella, Ribes, Agulló y Soldevilla, 2002), tal como la que se plantea en este proyecto en un contexto específico prioritario por su relevancia, como es el contexto escolar multicultural característico actualmente de nuestros centros educativos.

A nivel internacional, destaca la adopción de un nuevo enfoque de competencias (Boyatzis, 1982; Levy-Leboyer, 1997; Tejada 1999; Pereda y Berrocal, 1999, 2001; García, 2000, 2003), adoptado en políticas europeas de educación y formación (Tunning, 2003). Especial atención merece la comunidad de profesionales del ámbito de la investigación y de la práctica educativa The Collaborative for Academic, Social, and Emotional Learning (CASEL), fundada en EEUU por Goleman y Rockefeller Growald en 1994, cuyo ámbito de actuación es la enseñanza y el aprendizaje de las competencias socioemocionales. Este grupo trata de crear un cuerpo teórico sólido, contrastado en la práctica, y difundido a través de programas de formación de profesionales en competencias socioemocionales (Graczyk et al., 2000). Entre sus objetivos prioritarios destaca la revisión y evaluación de más de 250 programas para estimular el aprendizaje social y emocional en los centros educativos (CASEL, 2003).

Dentro del ámbito específico de la orientación intercultural, la fundación -en 1985- de la Association for Multicultural Counseling and Development, supuso un hito más hasta el punto de considerar actualmente que el multiculturalismo constituye la cuarta dimensión de la Orientación (complementaria a los enfoques psicodinámicos, conductistas y humanistas), dado que toda ayuda se origina en un contexto cultural. Sin embargo, las investigaciones sobre multiculturalidad suelen referirse ampliamente a la educación, no tanto a la orientación. Son muy diversos los grupos de investigación sobre la educación en contextos multiculturales, tanto a nivel internacional como español, desde la Sociedad Europea para la Educación Intercultural, Formación e Investigación (SIETAR), que pretende desarrollar y aplicar el conocimiento, valores y habilidades que permitan unas relaciones adecuadas entre distintas culturas, etnicas, grupos, organizaciones y niveles (www.sietar-europa.org/); el laboratorio de estudios interculturales de la Universidad de Granada; el Grupo Inter de la UNED que investiga, entre otros aspectos, el desarrollo de competencias interculturales en los orientadores y el profesorado, o la propia Red investigadora del 
Centro de Recursos para la Atención a la Diversidad Cultural en Educación (CREADERED) del MEC, con una sección específica dedicada a la interculturalidad.

\section{Conclusiones.}

Debido al crecimiento de los movimientos migratorios así como las respuestas insolidarias y racistas, junto con el fracaso académico de algunos colectivos, es preciso que toda la comunidad educativa se implique en el reto que supone el pluralismo cultural y las respuestas educativas correspondientes.

Desde el ámbito de la Orientación, y en respuesta a la situación multicultural de nuestro sistema educativo, es preciso señalar, en primer lugar, que a pesar de la existencia de los programas de intervención sobre competencias socioemocionales citados resulta difícil encontrar estudios publicados sobre la evaluación y eficacia de los mismos, así como investigaciones empíricas sobre programas de formación en competencias socioemocionales en contextos multiculturales. En consecuencia, parece pertinente continuar la línea de investigación de la profa. Repetto (2005-2007) aportando un programa en dichos contextos que, además de las repercusiones en el ámbito educativo, podría tener proyección internacional a través de los intercambios científicos con otros grupos de investigación, mediante artículos en publicaciones especializadas y ponencias en congresos.

Por tanto, se propone realizar una revisión actualizada del estado de la cuestión sobre las competencias socio-emocionales que favorecen la mejora del aprendizaje y la integración escolar y social de los alumnos de Educación Secundaria en contextos multiculturales, así como una evaluación de las necesidades de integración socio-educativas en contextos multiculturales; además se constata la necesidad de diseñar, validar, aplicar y evaluar un programa de intervención orientadora dirigido a desarrollar las citadas competencias de los alumnos de Educación Secundaria en contextos multiculturales.

Finalmente, se destaca que, tanto los procesos de inserción como las diversas transiciones profesionales exigen la propuesta de programas de Orientación, como por ejemplo el POCOSE, dirigidos al desarrollo de las competencias socioemocionales capaces de afrontar los efectos de la inestabilidad laboral, el desempleo, las exigencias de movilidad profesional, 
el complejo entramado de relaciones sociolaborales y la calidad de vida en el trabajo. De este modo, la Orientación Profesional superaría el desafío de convertirse en un auténtico recurso social para afrontar las múltiples elecciones y decisiones ocupacionales que cada individuo habrá de adoptar a lo largo de su vida.

\section{Referencias}

Álvarez, M. (2001). Diseño y evaluación de programas de educación emocional. Barcelona, Ciss-Praxis.

Arón, A.M., Milicia, N. (1996). Vivir con otros. Madrid, CEPE.

Bachman, J., Stein, S., Campbell, K. y Sitarenios, G. (2000). Emotional intelligence in the Collection of Debt. International Journal of Selection and Assessment, 8 (3), 176182).

Bar-On, R., Brown, J.M., Kirkcaldy, B.D. y Thomé, E.P. (2000): Emotional expression and implications for occupational stress, an application of the Emotional Quotient Inventory (EQ-i). Personality and Individual Differences, 28, 1107-1118 (2000).

Bisquerra, R. (2000). Educación emocional y bienestar. Barcelona, Praxis.

Bisquerra, R. (2004). Diseño, aplicación y evaluación de programas de educación emocional. En M.J. Iglesias (ed): El reto de la educación emocional en nuestra sociedad, 121161. A Coruña, Universidade da Coruña

Blasco, J.L., Bueno, V., Navarro, R., y Torregrosa, D. (2002). Programa de educación emocional. Propuestas para la tutoría. Valencia, Consejería de Cultura y Educación de la Generalitat valenciana

Boyatzis, R. E. (1982). The competent manager: A model for effective performance. Nueva York: John Wiley \& Sons.

Boyatzis, R.E., Goleman, D. y Rhee, K.S. (2000). Clustering competence in Emotional Intelligence. En Bar-On, R. y Parker, J.D. (Eds.), The competent manager: a model for effective performance. New York: Willey \& Sons.

Caruso, D.R. y Wolfe, Ch. J. (2001). Emotional intelligence in the workplace. En J. Ciarrochi, J. Forgas y J.D. Mayer (Eds), Emotional intelligence in everyday life. London: Psychology Press. 
Charbonneau, D. y Nicol, A.A.M. (2002): Emotional intelligence and prosocial behaviors in adolescents. Psychological Reports, vol. 90, 2, 361-370, abril 2002. (ResumenAbstract).

Chermiss, C. y Goleman, D. (2001). The emotionally intelligent workplace. San Francisco: Jossey-Bass.

Collaborative for Academic, Social and Emotional Learning (2003). Safe and sound: An educational leader's guide to evidence-based social and emotional learning programs. Chicago: Author.

De La Cruz y Mazaira (1997). Programa de Desarrollo afectivo. DSA. Madrid. TEA Ediciones.

Díaz-Aguado, M.J. (1996). Programas de educación para la tolerancia y prevención de la violencia en los jóvenes. Cuatro volúmenes y dos vídeos. Madrid: Instituto de la Juventud, Ministerio de Trabajo y Asuntos Sociales.

DiPerna, J.C. y Elliot, S.N. (1999). The development and validation of the academic competence evaluation scales. Journal of Psychoeducational Assessment, 17, 207-225.

Dryfoos, J.G. (1997). The prevalence of problem behaviors: Implications for programs. En R.P. Weissberg, T.P. Gullota, R.L. Hampton, B.A. Ryan, \& G.R. Adams (Eds). Healthy children 2010: Enhancing children's wellness, 17-46. Thousand Oaks, CA: Sage Publications.

Dulewicz, V. y Higgs, M.J. (1999). Can emotional intelligence be measured and developed? Leadership \& Organization Development Journal, 20 (5), 242-252.

Espejo, García-Salmones y Vicente (2000). El programa para el Desarrollo y Mejora de la Inteligencia Emocional. Madrid.

Extremera, N. y Fernández-Berrocal, P. (2004). El papel de la inteligencia emocional en el alumnado: evidencias empíricas, Revista Electrónica de Investigación Educativa, 6(2)

Fernández-Berrocal, P. y Extremera, N. (2002). La inteligencia emocional como una habilidad esencial en la escuela, Revista Iberoamericana de Educación, 29, 1-6.

Fernández-Berrocal, P., Extremera, N. y Ramos, N. (2004). Validity and reability of the Spanish modified version of the trait meta-mood scale, Psychological Reports, 94, 751755.

Filella, G., Ribes, R., Agulló, M.J. y Soldevilla, A. (2002). Formación del profesorado: asesoramiento sobre educación emocional en centros escolares de infantil y primaria, $E d u$ car, 30, 159-167. 
Garaigordobil, M. (2005). Diseño y evaluación de un programa de intervención socioemocional para promover la conducta prosocial y prevenir la violencia. Madrid: Centro de Publicaciones del Ministerio de Educación y Ciencia. Colección Investigación No 160 (Primer Premio Nacional de Investigación Educativa 2003).

García, M. (2000). Factores clave en el desarrollo de competencias. En Aguilló, E., Remeseiro, C. y Fernández, A.: Psicología del Trabajo, de las Organizaciones y de los Recursos Humanos. Madrid: Biblioteca Nueva.

García, M. (2003). Entrenamiento en competencias. Encuentros en Psicología Social, 1 (3), 27-32.

Goleman, D. (1996). Inteligencia Emocional. Barcelona, Kairos.

Graczyk, P.A., Weissberg, R.P., Payton, J.W., Elias, M.J., Greenberg, M.T. y Zins, J.E. (2000). Criteria for Evaluating the Quality of School-Based Social and Emotional Learning Programs. En R. Bar-On y J.D.A. Parker, The Handbook of Emotional Intelligence. Theory, Development, Assessment, and Application at Home, School, and in the Workplace, 391-410. San Francisco, Ca: Jossey-Bass.

Güell Barceló, M. y Muñoz Redón, J. (2000). Desconócete a ti mismo. Programa de alfabetización emocional. Barcelona, Paidos.

Güell Barceló, M. y Muñoz Redón, J. (2003). Educación emocional. Programa para la educación secundaria post-obligatoria. Barcelona, Praxis.

Guil, R. (2006). Adaptación socioescolar en contextos multiculturales: inteligencia emocional y estrategias de aculturación exitosas. Proyecto I+D financiado por el MEC para el período 2007-2009.

Hawkins, J.D., Farrington, D.P. y Catalano, R.F. (1998). Reducing violence through the schools. In D.S. Eliot, B.A. Hamburg \& K.R. Williams (Eds.), Violence in American schools: a new perspective, 188-216. Cambridge: Cambridge University Press.

Haynes, N.M., Ben-Avie, M. y Ensign, J. (2003). How social and emotional development add up: getting results in math and science education. New York: Teachers College Press.

Hettich, P. (2000). Transition processes from college to career. Washington: APA.

Ibarrola, B. (2004). La educación de la inteligencia emocional, Actas de las IX Jornadas técnicas: otras formas de educar. Madrid.

Ibarrola, B. y Delfo, E (2003a). Sentir y pensar. Programa de Inteligencia emocional para niños de 3 a 5 años. Madrid: SM

Ibarrola, B. y Delfo, E (2003b). Sentir y pensar. Programa de Inteligencia emocional para niños de 6 a 8 años. Madrid: SM 
Izquierdo, C. (2001). Comunicación grupal y desarrollo personal: las emociones en el punto de mira de la inteligencia y el aprendizaje organizacional. En Del Rincón, B. (Coord.), Presente y futuro del trabajo psicopedagógico. Madrid: Ariel Educación.

Levy-Leboyer, C. (1997). Gestión de las competencias: cómo analizarlas, cómo evaluarlas, cómo desarrollarlas. Barcelona: Gestión 2000.

López Cassà, E. (2003). Educación emocional. Programa para 3-6 años. Barcelona, Praxis.

Malecki, C.K. y Elliott, S.N. (2002). Children's social behaviours as predictors of academic achievement: a longitudinal analysis. School Psychology Quarterly, 17 (1), 1-23.

Ministerio de Educación y Ciencia (1997). Convivir es vivir. Programa de desarrollo de la convivencia en centros educativos de la Comunidad de Madrid. Madrid: Defensor del Menor en la Comunidad de Madrid.

Ortega, J.A. (1996). Educación Multicultural para la Tolerancia y la Paz. Fundamentos y estrategias didácticas. Granada: Grupo Editorial Universitario

Parker, J. D. A., Summerfeldt, L. J., Hogan, M. J. y Majeski, S. A. (2004). Emotional intelligence and academic success: examining the transition from high school to university. Personality and Individual Differences, 36 (1), 163-172.

Pascual, V. y Cuadrado, M. (2001). Educación emocional. Programa de actividades para E.S.O. Barcelona, CissPraxis.

Pasi, R.J. (2001). Higher expectations: promoting social emotional learning and academic achievement in your school. New York: Teachers College Press.

Pena, E. y Repetto, E. (2006). Propuestas educativas para el desarrollo de la inteligencia emocional, Revista de Educación (pendiente de publicación).

Pereda, S. y Berrocal, F. (1999). Gestión de Recursos Humanos por Competencias. Madrid: Editorial Centro de Estudios Ramón Areces.

Pereda, S. y Berrocal, F. (2001). Técnicas de gestión de recursos humanos por competencias. Madrid: Editorial Centro de Estudios Ramón Areces.

Petrides, K.V., Frederickson, N., y Furnham, A. (2004). The role of trait emotional intelligence in academic performance and deviant behavior at school. Personality and Individual Differences, 36, 277-293.

Renom, A. (2003). Educación emocional. Programa para la educación primaria. Barcelona, Praxis.

Repetto, E. (2005). Proyecto I+D: “Orientación y desarrollo de competencias socioemocionales a través de las prácticas en empresa", financiado por el Ministerio de Educación, SEJ2004-07648/EDUC. 
Repetto, E. (2006a). Las competencias socioemocionales y el fracaso escolar. Conferencia pronunciada en las I J Jornadas Pozuelo Alarcón, Madrid

Repetto, E. (2006b). Orientación intercultural e inclusión social. Actas de la Conferencia Internacional Orientación, Inclusión Social y Desarrollo de la Carrera, 41-53.

Repetto, E., Beltrán, S, Garay-Gordovil, A. y Pena, M. (2006). Validación del Inventario de competencias socioemocionales -importancia y presencia- (ICS-I; ICS-P) en estudiantes de ciclos formativos y de Universidad, Revista Española de Orientación y Psicopedagogía 17 (2), 213-223.

Repetto, E. y Pérez-González, J.C. (en prensa). Formación en competencias socioemocionales a través de las prácticas en empresas. Revista Europea de Formación ProfesionalEuropean Journal of Vocational Training, 40 (January-April 2007/I). Monográfico especial sobre competencias.

Repetto, E. y Pena, M. (2006a). Orientación intercultural, respuesta al fenómeno de la inmigración, (pendiente de publicación)

Repetto, E. y Pena, M. (2006b). Cross-cultural counselling and social inclusion in Spain, Paradigma, vol. 27, 2 (en prensa)

Salmurri, F. y Blanxer, N. (2002). Programa para la educación emocional en la escuela. En R. Bisquerra, La práctica de la orientación y la tutoría,145-179. Barcelona, Praxis.

Salvador, M. (2000). Programa de desarrollo emocional. Málaga, Aljibe.

Scales, P.C. y Leffert, N. (1999). Developmental assets: A synthesis of the scientific research on adolescent development. Minneapolis, Search Institute.

Schutte, N.S., Malouff, J. M., Bobik, Ch., Coston, T. D., Greeson, C., Jedlicka, Ch., Rhodes, E. y Wendorf, G. (2001). Emotional Intelligence and Interpersonal Relations. The Journal of Social Psychology, 141 (4), 523-536.

Segal, J. (2002). Good leaders use "emotional intelligence". Emotionally intelligent leadership is a skill that can be learned and taught throughout life. Health Progress, 83 (3), 44-46).

Steiner, C. (1998). La educación emocional. Buenos Aires, Avon Books.

Tejada, J. (1999). Acerca de las competencias profesionales. Herramientas, 56, 20-30.

Tornos, A y Aparicio, R. (2005). Las redes sociales de los inmigrantes en España: un estudio sobre el terreno. Madrid. Ministerio de Trabajo y Asuntos Sociales. Asuntos Sociales.

Torres, J.C. (1996). Estrategias de aprendizaje: la tercera vía, Comunidad educativa, 230, 5-8 (cuadernillo central). 
Traveset, M. (1999). Educación emocional: estrategias de intervención, Aula de Innovación Educativa, 89, pp. 15-20.

Vallés, A. (2003). Emoción-ate con inteligencia. Valencia: Promolibro.

Vallés A. y Vallés C. (2000). Inteligencia emocional. Aplicaciones educativas. Madrid, EOS.

Vallés, A. y Vallés, C. (1999). Desarrollando la Inteligencia emocional. Programa para el desarrollo de la inteligencia emocional I. Educación Primaria (ciclo $1^{\circ}$ ). Madrid: EOS.

Weissberg, R.P. y Greenberg, M.T. (1998). School and community competence-enhancement and prevention programs. En I.E. Sigel \& K.A. Renninger (Eds), Handbook of child psychology: Vol 4. Child psychology in practice (5a ed., pp.877-954). Nueva York, John Wiley \& Sons.

Wong, C.S.; Law, K.S. (2002). The effects of leader and follower emotional intelligence on performance and attitude: An exploratory study, Leadership Quarterly, 13, pp. 243274.

Zeidner, M., Roberts, R. D., y Matthews, G. (2002). Can emotional intelligence be schooled? A critical review. Educational Psychologist, 37 (4), 215-231.

Zins, J. E. y Weissberg, R. P., Wang, M. C. y Walberg, H. J. (2004). Building academic success on social and emotional learning: What does the research say? New York, Teachers College Press. 